\title{
Integration of Frequency Domain Wideband Antenna Nulling and Wavenumber Domain Image Formation for Multi-Channel SAR
}

\author{
M. Bucciarelli, ${ }^{1}$ D. Pastina, ${ }^{1}$ D. Cristallini, ${ }^{2}$ M. Sedehi, ${ }^{3}$ and P. Lombardo ${ }^{1}$ \\ ${ }^{1}$ DIET Department, University of Rome "La Sapienza", Via Eudossiana 18, 00184 Rome, Italy \\ ${ }^{2}$ Fraunhofer FHR, Department of PSR, Fraunhoferstrasse 20, 53343 Wachberg, Germany \\ ${ }^{3}$ Finmeccanica S.p.A., Via Tiburtina Km 12400, 00131 Rome, Italy \\ Correspondence should be addressed to M. Bucciarelli; mbucciarelli@infocom.uniromal.it
}

Received 30 October 2015; Revised 25 January 2016; Accepted 28 January 2016

Academic Editor: Wei Liu

Copyright (c) 2016 M. Bucciarelli et al. This is an open access article distributed under the Creative Commons Attribution License, which permits unrestricted use, distribution, and reproduction in any medium, provided the original work is properly cited.

A Multichannel Synthetic Aperture Radar (M-SAR) exploiting an antenna nulling based Electronic Counter-Counter Measures (ECCM) technique shall be able to cancel the effects of noise-like interferences over the collected SAR data. Since SAR systems often work with wide bandwidths to provide high resolution images, ECCM technique must account for the presence of wideband interference signal. In this paper we consider a wideband antenna nulling technique based on space-frequency adaptive nulling and we propose an integration of the WB antenna nulling scheme within the focusing algorithm for M-SAR systems, thus allowing a fusion between ECCM and usual SAR processing steps. The computational cost of the integrated algorithm is compared with the cost of more traditional sequence of the wideband extension of the Side-Lobe Canceller and the focusing operation, to show the computational feasibility of the proposed integrated algorithm. The possibility to perform suboptimally the space-frequency adaptive nulling is also considered.

\section{Introduction}

The effect of a wideband (WB) noise-like interference for imaging radars is to mask the scene visible in the imaged area with a high uniform noise level [1-3]. In order to assure the typical functionality of a Synthetic Aperture Radar (SAR) system, a strategy to suppress the undesired component of the received signal must be identified and implemented, with the requirement to be suitable for the subsequent SAR processing.

Radar systems based on antenna arrays can typically employ different strategies to protect themselves from unwanted intentional or environmental electromagnetic interferences, especially when impinging on the side-lobes of the synthesized array pattern. A basic way to try and mitigate the effects of undesired components of the received signal is to lower the level of the side-lobes of the array pattern [4]: different strategies can be employed, from traditional amplitude tapering [5] to efficient antenna aperture thinning at element or subarray level, as for example, the one developed and implemented in $[6,7]$. A different way to reject the interference component from the received signal is to null the synthesized pattern in the estimated jammer Direction of Arrival (DoA) performing the adaptive processing called antenna nulling. The typical formulation of the problem $[4,8]$ accounts for a monochromatic interference waveform impinging on the array antenna from directions sufficiently different from the main beam steering and exploits either the fully adaptive configuration, or the SideLobe Canceller (SLC) scheme or the multiple-beam/multiplechannel/subarray based adaptive processing. This last configuration is particularly interesting since it helps making adaptivity practically feasible for systems where array of hundreds or thousands of elements are involved at the cost of an affordable reduction of the available degrees of freedom.

From the previous considerations the exploitation of an array based M-SAR along with an adaptive antenna nulling technique shall be able to cancel the effects of WB continuous wave interferences over the collected SAR data, thus 
fundamentally allowing the normal SAR operation. However, since SAR systems often work with wide bandwidths to provide high resolution images, the ECCM technique must account for the presence of WB interference signals. The usual narrowband (NB) nulling techniques based on adaptive beamforming might not be effective to counteract WB interferences; therefore, specific WB techniques have to be considered.

In the literature several WB nulling techniques have been proposed, starting from different approaches. First of all it can be proved that a WB jammer is equivalent to an infinite number of monochromatic waves impinging on the antenna array from different angular directions near the nominal one: strategies for WB nulling could imply widening of the angular interval where the jammer is rejected by imposing multiple narrowband nulls around the jammer nominal DoA or by flattening the narrowband null constraining the derivatives of the adaptive pattern around the jammer DoA [9]. It is apparent how the rationale to allow WB nulling is increasing the number of available degrees of freedom, making the array transfer function as seen by the jammer frequency dependent [8]. The traditional and most investigated approach to achieve this goal is using tapped delay lines (TDLs) for each receiving channel $([8,10-14]$, and in particular for M-SAR in [15]). A typical extension of this fast Space-Time Adaptive Processing (fast STAP) approach is represented by the SLC architecture [15-17], where the use of a single sample from the main channel largely helps in keeping limited the impact of the wideband jammer cancellation processing on the range impulse response. Some wideband nulling algorithms work in the frequency domain operating a Fourier transform on each set of fast-time samples collected on the available receiving channels, resulting in a subbanding operation that allows the applications of traditional narrowband nulling techniques in each subband. In particular, in $[8,18]$ the equivalence of the fast STAP and the space-frequency adaptive processing is proven. It has to be underlined that frequency subbanding has been widely considered also in traditional STAP schemes for ground moving target indication (GMTI). Evolutions of these schemes to limit the computational burden have been considered $[19,20]$, allowing the focusing of all the subband into a single reference subband where narrowband STAP can be performed: however, this approach seems to be not suited for high resolution imaging due to the induced range resolution reduction. In [21-24] the possibility of performing WB jammer cancellation in the frequency domain is mentioned and the concept of wideband nulling after stretch processing is presented. Depending on the relation between the time duration of the transmitted chirp and the maximum range of interest for the considered radar sensor, deramping followed by low-pass filtering can result in a lower bandwidth and a narrowband nulling technique could follow. Typically in SAR imaging the better is the range resolution the smaller is the range swath and the stretch processing could be considered a viable solution to form high resolution range profiles; however, it is always possible to process the acquired SAR data according to focusing algorithms exploiting the traditional matched filtering approach and working in the wavenumber domain, as the Range Migration Algorithm (RMA), [25], or the Range Doppler Algorithm, [26].

In this paper the main idea is to apply the frequency domain WB cancellation algorithm based on subbanding to the whole set of samples collecting each Pulse Repetition Time (PRT) in the range domain to counteract side-lobe noise-like jammers, thus allowing an integration of the antenna nulling in the frequency domain with a wavenumber domain focusing technique for M-SAR. Indeed the batch characteristic of the proposed nulling algorithm in the frequency domain is not a drawback, since the SAR image is formed after receiving all pulses anyway. This approach is different from the typical way to process the incoming signal to recover the normal imaging capability despite the presence of the interference, which is to perform a WB antenna nulling technique (i.e., WB-SLC) followed by the chosen focusing algorithm. The basic concept of the proposed technique was presented in [27]: from this starting point this paper moves on detailing a much accurate formalization of the problem, presenting results on different and more challenging simulated scenarios and finally providing a new and full analysis on the computational cost of the proposed algorithm compared with the burden of the more traditional sequence of WBSLC and focusing, also taking into account the possibility of suboptimal implementations and their impacts on the SAR pulse response and on the nulling performance.

In [27] a very rough and draft model for the received signal have been presented, instead here a detailed signal model is shown allowing the subsequent formalization of the wideband nulling: starting from the general and wellknown fast-time STAP, where nulling weights are achieved based on the Least Mean Square criterion, and from the WB-SLC scheme, the proposed wideband nulling scheme in the frequency domain is presented. In detail it starts operating a Fourier transform (subbanding) on each set of fast-time samples collected on the available receiving channels during a PRT, as the most of the focusing algorithms in the wavenumber domain do. Adaptive coefficients must be applied at this stage to the collected data. The derivation of the expression of the weights to be applied for wideband nulling in the time domain shows that, as it will be clear in the following, the WB adaptive coefficients do not depend on the steering vector at carrier frequency as in the NB case but on the specific samples of the transmitted signal, thus nulling and matched filtering appears to be fused together. Indeed, the structure of the adaptive weight vector to be applied in the frequency domain is explicitly derived, differently from [27] where it was only shown, proving the ability of the proposed algorithm of splitting the procedures of jammer rejection and matched filtering [28, 29], otherwise fused in the space-fast-time nulling weights. Therefore, after Fourier transform traditional NB nulling can be applied in each subband obtaining an equivalent single-channel data set free of the interference after recombination. From this point the subsequent steps of a standard wavenumber focusing processing (including range compression) can follow. This integration between nulling and focusing techniques proves to allow an effective cancellation of the WB interference independently on the jammer equivalent isotropic radiated 
power (EIRP); differently from [27] where a side-lobe jammer with a high level EIRP was added to a very low noise contribution, two disturbance scenarios are investigated in the performance analysis section, where a single side-lobe jammer with two different levels of EIRP add to a severe noise contribution. In both cases jammer appears to be rejected by the proposed integrated wideband nulling and focusing technique potentially recovering the computational cost with respect to the traditional cascade of WB-SLC and focusing depending on the interference characteristics. Indeed in the time domain the number of fast-time samples to be processed or equivalently the length of the TDLs to be employed for an effective nulling of the wideband interferer obviously depends on its strength. Therefore, a balance point can be found where a batch processing as the one performed in the WB frequency nulling fused with the focusing algorithm begins to have a computational cost comparable with the cascade of SLC based WB nulling and image formation. To highlight, confirm, and prove this core potentiality just hinted in [27], in this paper an accurate derivation of the computational costs of the proposed integrated WB nulling and RMA is derived and compared with the cost of a more traditional sequence of WB-SLC and RMA, chosen as a reference wavenumber domain focusing algorithm. Moreover, suboptimal implementations of the frequency WB nulling technique are here considered, either widening the extent of each subband or reducing the number of updates of the adaptive coefficients, which nominally occur at each PRT. This analysis definitely expands what is presented in [27], where only the widening of frequency subbands was considered, and allows us to add an important consideration: results show how the impact of the first suboptimal approach is limited, thus allowing us to save computational cost even for jammers of high intensity, while the number of updates of the nulling weights is critical.

The paper is organized as follows: in Section 2 the system and the signal models are described, while in Section 3 the general expression of the space-fast-time weights for WB nulling is derived using the Least Mean Square (LMS) approach, followed by the presentation of the WB space-frequency adaptive nulling. The integration of the last technique with a wavenumber domain focusing algorithm is presented in Section 4. Section 5 follows with some simulated results. In Section 6 the computational cost of the integrated algorithm is compared with the cost of the more traditional sequence of WB-SLC and focusing, to show the computational feasibility of the proposed integrated algorithm; moreover, suboptimal versions of the frequency domain WB nulling algorithm are investigated to lower its computational cost in Section 7. Finally some conclusions are drawn in Section 8.

\section{System and Signal Model Description}

In our analysis the SAR antenna is an active phased array formed by $N_{\mathrm{el}}$ isotropic elements arranged to provide a number $C$ of receiving channels, each connected to a different beam.
The considered M-SAR transmits a WB waveform $s(t)$ (as a chirp) of length $T_{p}$ and bandwidth $B$ every PRT during the acquisition period.

The signal received by the $p$ th radiating element of the array is the superposition of a useful part and a disturbance component due to the jammer and thermal noise; the first is supposed to be a wideband noise-like signal and the last is independent at each element in the array and almost decorrelated over the bandwidth $B$. The useful component of the signal received by the $p$ th element in the array is an echo of the transmitted signal: this echo is attenuated by a factor depending on the link-budget and delayed according to the two-way distance of a generic scatterer in the scene with DoA $\left(\theta_{i}, \varphi_{i}\right)$ from the $p$ th radiating element. On the other hand, the signal received from a jammer with DoA $\left(\theta_{j}, \varphi_{j}\right)$ is attenuated and delayed depending on the oneway distance of the jammer from the $p$ th element. It has to be noticed that the useful component of the received signal suffers from a two-way attenuation and the jammer only from a one-way propagation loss. Therefore, even if the interference transmitted power is not high and it is received by the side-lobes of the SAR antenna, it could deny the SAR imaging capability.

Beamforming and steering operations are performed to pass from $N_{\mathrm{el}}$ radiating elements to $C$ receiving channels: for the ch channel $(\mathrm{ch}=0, \ldots, C-1)$ the received signal can be written as

$$
x_{r x}^{\mathrm{ch}}\left(t, \theta_{i}, \varphi_{i}, \theta_{j}, \varphi_{j}\right)=s_{r x}^{\mathrm{ch}}\left(t, \theta_{i}, \varphi_{i}\right)+x_{d}^{\mathrm{ch}}\left(t, \theta_{j}, \varphi_{j}\right),
$$

where $s_{r x}^{\mathrm{ch}}\left(t, \theta_{i}, \varphi_{i}\right)$ is the useful component, while

$$
x_{d}^{\mathrm{ch}}\left(t, \theta_{j}, \varphi_{j}\right)=j^{\mathrm{ch}}\left(t, \theta_{j}, \varphi_{j}\right)+n^{\mathrm{ch}}(t)
$$

is the disturbance depending on jammer and noise, respectively.

It is well known that the number of available degrees of freedom in the array should be increased to cancel out the WB jammer; if a limited number of receiving channels is available, then a set of fast-time samples could be taken at each channel and processed according to a proper space-fasttime scheme in order to obtain a WB antenna nulling [810]. Figure 1 shows a possible processing scheme, where the space-fast-time samples are properly weighted to produce the output jammer-free signal. As a consequence the following vector representation for the received data at channel level is considered.

Describing the signal as a function of slow-time variable $n=\lfloor t /$ PRT $\rfloor+1$ and the fast-time variable $\hat{t}=t+(n-1)$. PRT and omitting the explicit dependence from the DoAs, the received signal in (1) is given by

$$
\mathbf{x}_{r x}\left(n, \widehat{t}_{l}\right)=\mathbf{s}_{r x}\left(n, \widehat{t}_{l}\right)+\mathbf{x}_{d}\left(n, \widehat{t}_{l}\right),
$$

where $\widehat{t}_{l}$ is the $l$ th sample of $\widehat{t}$.

These vectors are organized as stacked column vectors of size $C \cdot K \times 1$, where $K$ is the number of signal samples spaced 


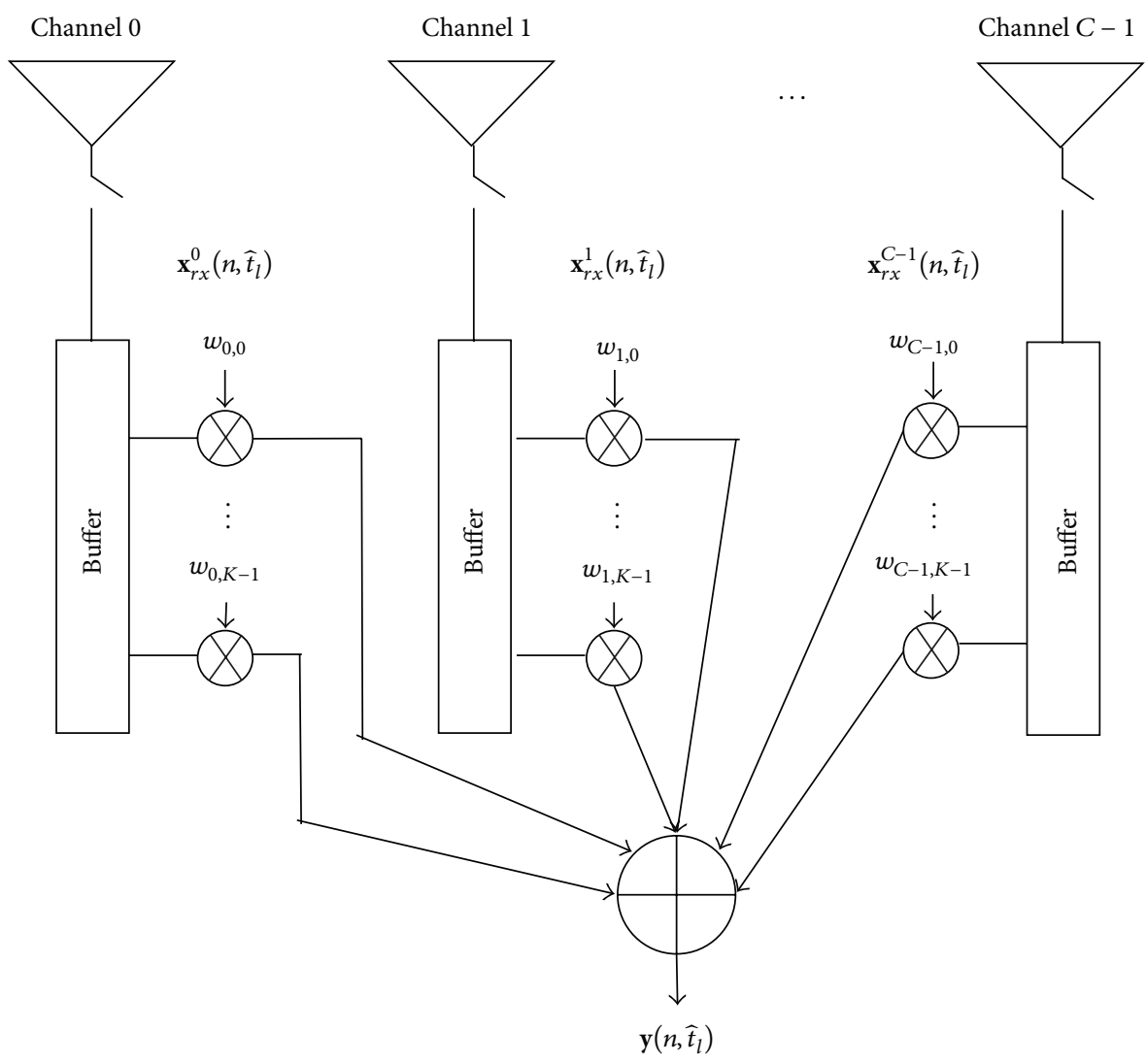

FIGURE 1: Space-fast-time processing.

by the sample period $T_{s}$ buffered for each channel in the array at the fast-time instant $\widehat{t}_{l}$ :

$$
\begin{aligned}
& \mathbf{s}_{r x}\left(n, \widehat{t}_{l}\right) \\
& =\left[\begin{array}{llllll}
\mathbf{s}_{r x, 0}\left(n, \widehat{t}_{l}\right) & \cdots & \mathbf{s}_{r x, k}\left(n, \widehat{t}_{l}\right) & \cdots & \mathbf{s}_{r x, K-1}\left(n, \widehat{t}_{l}\right)
\end{array}\right]^{T} \\
& \text { with } \mathbf{s}_{r x, k}\left(n, \widehat{t}_{l}\right) \\
& =\left[s_{r x}^{0}\left(n, \widehat{t}_{l}-k T_{s}, \theta_{i}, \varphi_{i}\right) \cdots s_{r x}^{C-1}\left(n, \widehat{t}_{l}-k T_{s}, \theta_{i}, \varphi_{i}\right)\right] \\
& \mathbf{x}_{d}\left(n, \widehat{t}_{l}\right) \\
& =\left[\begin{array}{lllll}
\mathbf{x}_{j, 0}\left(n, \widehat{t}_{l}\right) & \cdots & \mathbf{x}_{j, k}\left(n, \widehat{t}_{l}\right) & \cdots & \mathbf{x}_{j, K-1}\left(n, \widehat{t}_{l}\right)
\end{array}\right]^{T}+\mathbf{n}
\end{aligned}
$$

with $\mathbf{x}_{j, k}\left(n, \widehat{t}_{l}\right)$

$$
=\left[x_{j}^{0}\left(n, \widehat{t}_{l}-k T_{s}, \theta_{j}, \varphi_{j}\right) \cdots x_{j}^{\mathrm{C}-1}\left(n, \hat{t}_{l}-k T_{s}, \theta_{j}, \varphi_{j}\right)\right] .
$$

It has to be underlined that, if the signal DoA corresponds to the steering direction $\left(\theta_{0}, \varphi_{0}\right)$, each element of the vector $\mathbf{s}_{r x, k}\left(n, \widehat{t}_{l}\right)$ will be a version of the transmitted waveform properly delayed according to the lag $\tau\left(\theta_{0}, \varphi_{0}\right)$ and scaled according to the link-budget factor $A\left(\theta_{0}, \varphi_{0}\right)$ and the gain $G_{r x}^{\mathrm{ch}}\left(\theta_{0}, \varphi_{0}\right)$ of the $r x$ formed beams independently from the frequency. Therefore, defining the gain vector as

$$
\boldsymbol{\sigma}=\left[\begin{array}{llll}
G_{r x}^{0}\left(\theta_{0}, \varphi_{0}\right) & \cdots & G_{r x}^{C-1}\left(\theta_{0}, \varphi_{0}\right)
\end{array}\right],
$$

the desired part of the received signal at channel level could be written as

$$
\mathbf{s}_{r x}\left(n, \widehat{t}_{l}\right)=A\left(\theta_{0}, \varphi_{0}\right) \cdot\left[\mathbf{s}\left(n, \widehat{t}_{l}\right) \otimes \boldsymbol{\sigma}\right]^{T},
$$

where $\mathbf{s}\left(n, \widehat{t}_{l}\right)$ is a row vector containing $K$ samples of the transmitted waveform and $\otimes$ represent the Kronecker product.

\section{Nulling Approaches for WB Signals}

As seen before, a SAR system typically works with wide bandwidths to obtain high resolution in the range dimension and also the protection scheme must account for the presence of a WB interference signal.

In order to perform wideband antenna nulling (Figure 1) over the received data at channel level, a proper weight vector must be determined. We assume that the desired signal is deterministic and decorrelated from the interference and the thermal noise. In this case applying a LMS criterion when the DoA of the desired signal corresponds to the steering direction, the following weight vector is obtained $[8,10,11$, 29]:

$$
\mathbf{w}\left(n, \widehat{t}_{l}\right)=\mathbf{Q}_{d}^{-1}(n) \mathbf{s}_{0}\left(n, \widehat{t}_{l}\right) .
$$




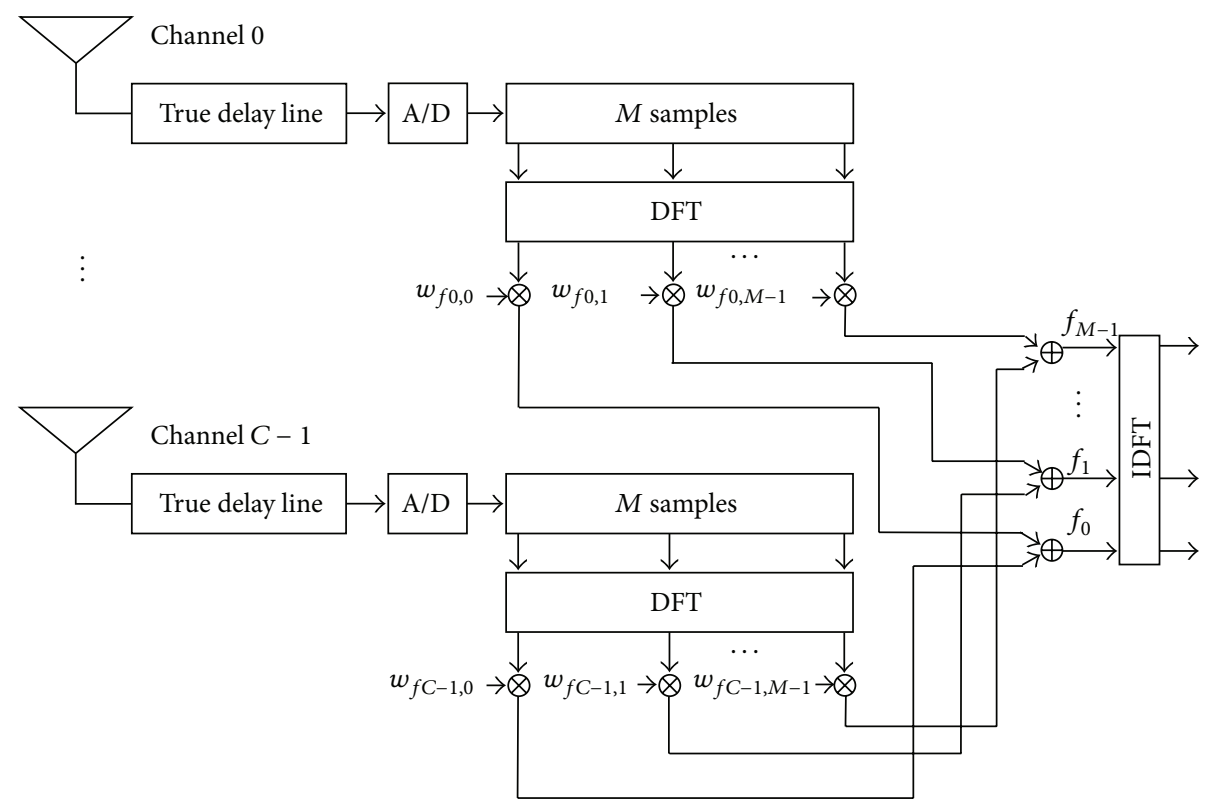

FIGURE 2: WB nulling in the frequency domain.

$\mathbf{Q}_{d}(n)$ is the $C \cdot K \times C \cdot K$ space-fast-time covariance matrix of the received interference plus noise and $\mathbf{s}_{0}\left(n, \widehat{t}_{l}\right)$ is the reference signal vector of size $C \cdot K \times 1$ defined as

$$
\mathbf{s}_{0}\left(n, \widehat{t}_{l}\right)=\left[\mathbf{s}\left(n, \widehat{t}_{l}\right) \otimes \boldsymbol{\sigma}\right]^{T}
$$

Unlike the narrowband case, $\mathbf{s}_{0}\left(n, \widehat{t}_{l}\right)$ is not simply the spatial steering vector, but it depends on the specific samples of the transmitted waveform. This makes it largely timevarying, since the two operations of antenna nulling and pulse compression are necessarily considered together by the selected criterion. An additional drawback of this approach is that the use of tapped delay lines leads necessarily to a temporal filtering of the incoming signal and thus a distorted version of the SAR point spreads function.

To try and overcome these issues the WB extension of the SLC processing can be taken into account. It can be directly derived from Figure 1 considering that there are one main channel (MC) and C-1 Auxiliary Channels (ACs) and that for each output sample only a single received signal sample from the MC is processed, while a set of $K$ samples is considered for each AC. Here we recall that the use of a single sample from the main channel largely helps in splitting the pulse compression from the wideband cancellation and allows keeping limited the impact of the cancellation processing on the range impulse response. Assuming the standard SLC approximation (auxiliary channels gain much lower than the main channel gain in the antenna steering direction), the weight vector $\mathbf{w}\left(n, \widehat{t}_{l}\right)$ becomes [29]

$$
\mathbf{w}_{\mathrm{SLC}}(n)=\mathbf{Q}_{d \mathrm{SLC}}^{-1}(n) \mathbf{e}_{1} \mathbf{s}_{0}\left(n, \widehat{t}_{0}\right) \approx \mathbf{Q}_{d \mathrm{SLC}}^{-1}(n) \mathbf{e}_{1},
$$

where $\mathbf{e}_{1}=\left[\begin{array}{llll}1 & 0 & \cdots & 0\end{array}\right]^{T}$, the dependence of the weight vector on the specific sample of reference signal is negligible especially for side-lobe jammers [29], and the disturbance covariance matrix is defined as

$$
\mathbf{Q}_{d S L C}(n)=\left[\begin{array}{cc}
q_{d, M}(n) & \mathbf{q}_{d, M A}(n) \\
\mathbf{q}_{d, M A}^{H}(n) & \mathbf{Q}_{d, A}(n)
\end{array}\right]
$$

$q_{d, M}(n)$ is the autocovariance of the main channel, $\mathbf{q}_{d, M A}$ vector of size $1 \times(C-1) \cdot K$ is the covariance between the main and the auxiliary channels, and $\mathbf{Q}_{d, A}$ is a Toeplitz and Hermitian block covariance matrix for the auxiliary channels of size $(C-1) \cdot K \times(C-1) \cdot K$.

An alternative way to adaptively null wideband interferences is through NB nulling applied after subbanding. In particular, Figure 2 shows the proposed frequency domain processing scheme for WB antenna nulling; instead of considering a sliding process performed over $K$ signal samples, a DFT is applied over a batch of $M$ samples, for example, obtained at Nyquist sampling rate from all range acquisition area. We assume that a previous operation of demodulation has been performed along with the steering of the beams pertaining to the different receiving channels through true delay lines. A linear transformation, as a DFT, of the received signal samples of each receiving channel does not affect the optimum structure for the weight determination as in (7) [18]. It also does not affect the performance of the optimum cancellation scheme. It has been shown in the past that if $M=$ $K$ and the same sampling distance is considered, the same performance in interference cancellation is obtained with a frequency domain approach and a traditional time domain approach $[8,18]$.

Since the algorithm operates over a batch of received samples, signal and weight vectors no longer depend on the particular fast-time instant $\widehat{t}_{l}$, which will be omitted in the following. 
The weight vector in the frequency domain becomes

$$
\mathbf{w}(n, f)=\mathbf{Q}_{d f}^{-1}(n) \mathbf{s}_{0}(n, f),
$$

where $\mathbf{s}_{0}(n, f)$ is the reference signal vector of size $M \cdot C \times 1$ in the frequency domain and $\mathbf{Q}_{d f}(n)$ is the interference plus noise spectral matrix of size $M \cdot C \times M \cdot C$.

Assuming a white interference in band $B$ and supposing we can realize the DFT transformation on a very large sample set (theoretically infinite) at Nyquist rate the different spectral components of the interference are decorrelated [28]; the same result is also valid for the thermal noise filtered in band $B$, apart from the natural decorrelation between the different antenna element contributions. Under these hypotheses the spectral matrix $\mathbf{Q}_{d f}(n)$ is a diagonal block matrix and the weight vector $\mathbf{w}(n, f)$ can be written as

$$
\mathbf{w}(n, f)=\left[\begin{array}{cccc}
\left(\mathbf{Q}_{d f}^{-1}(n, 0) \boldsymbol{\sigma}\right) & \mathbf{0} & \cdots & \mathbf{0} \\
\mathbf{0} & \left(\mathbf{Q}_{d f}^{-1}(n, 1) \boldsymbol{\sigma}\right) & \cdots & \mathbf{0} \\
\vdots & \vdots & \ddots & \vdots \\
\mathbf{0} & \mathbf{0} & \cdots & \left(\mathbf{Q}_{d f}^{-1}(n, M-1) \boldsymbol{\sigma}\right)
\end{array}\right] \mathbf{s}(f, n)=\mathbf{M}_{\mathbf{w}}(n) \mathbf{s}(f, n),
$$

where $\mathbf{Q}_{d f}^{-1}(n, k)$ is the inverse of the undesired signal spectral matrix block of size $C \times C$ pertaining to the $k$ th spectral component:

$$
\mathbf{Q}_{d f}(n, k)=\left[\begin{array}{cc}
q_{d f, M}(n, k) & \mathbf{q}_{d f, M A}(n, k) \\
\mathbf{q}_{d f, M A}^{H}(n, k) & \mathbf{Q}_{d f, A}(n, k)
\end{array}\right],
$$

where $q_{d f, M}(n, k)$ is a scalar, $\mathbf{q}_{d f, M A}(n, k)$ is a vector of size $1 \times$ $(C-1)$, and $\mathbf{Q}_{d f, A}(n, k)$ is the spectral matrix for the auxiliary channels of size $(C-1) \times(C-1)$.

Equation (12) shows that in the frequency domain it is possible to apply a spatial-only cancellation filter independently at each spectral component. This is encoded by the $(C \cdot M \times M)$ block-diagonal matrix $\mathbf{M}_{\mathbf{w}}(n)$. Moreover, the matched filtering in the frequency domain is encoded in the vector product with vector $\mathbf{s}(n, f)$ representing the transmitted signal in the frequency domain and it can be applied separately from the cancellation stage. As it is apparent the knowledge of the signal waveform is not required anymore for the wideband cancellation and the $k$ th component of the weight vector used for the nulling purpose becomes

$$
\mathbf{w}\left(n, f_{k}\right)=\mathbf{Q}_{d f}^{-1}(n, k) \cdot \boldsymbol{\sigma} .
$$

If it occurs that the gain of one channel (e.g., the first) is higher than the gain of the others in the steering direction (as for the WB-SLC) we can write the weight vector as follows:

$$
\mathbf{w}\left(n, f_{k}\right) \approx \mathbf{Q}_{d f}^{-1}(n, k) \mathbf{e}_{1} G_{M}\left(\theta_{0}, \phi_{0}\right)
$$

with $\mathbf{e}_{1}=\left[\begin{array}{llll}1 & 0 & \cdots & 0\end{array}\right]^{T}$, and $G_{M}\left(\theta_{0}, \phi_{0}\right)$ is the gain in the steering direction of the main channel.

\section{Integrated WB Nulling in the Frequency Domain and Focusing Algorithm}

A typical way to process the incoming signal to recover the normal imaging capability despite the presence of the interference is to perform a WB antenna nulling technique followed by the chosen focusing algorithm. The characteristics of the described space-frequency adaptive processing suggest another option, that is, the integration of WB antenna nulling and focusing technique; moreover, the batch characteristic of the proposed nulling algorithm in the frequency domain is not a drawback, since the SAR image is formed after receiving all pulses. Since the proposed WB nulling technique operates a DFT on each set of fast-time samples collected on the available receiving channels, the main idea is to apply the cancellation algorithm to the whole set of samples collected by the SAR in the range domain at each PRT (space-frequency adaptive processing), thus allowing an integration of the antenna nulling with a focusing technique working in the wavenumber domain, like, for example, the Range Migration Algorithm (RMA) [25] or the Range Doppler Algorithm (RDA) [26]. Figure 3 shows the block diagram of the resulting integrated algorithm. More specifically for each PRT, that is, for each slow-time instant of the SAR acquisition, received data sampled at Nyquist rate from each one of the $C$ channels are Fourier transformed providing $M$ independent spectral components. Therefore, data can be arranged as $M$ spectral vectors of $C$ samples and therefore they can be processed according to the wideband nulling technique in the frequency domain as discussed in Section 3 (black dashed box in the figure), providing as output a single-channel jammer-free vector, with a sample for each spectral subband. After the collection of these vectors for all the slow-time instant in the acquisition the remaining steps of the chosen SAR data processing follow (red dashed box in the figure, one for RMA and one for RDA).

\section{Performance Analysis}

To prove the effectiveness of the proposed technique, we considered the following scenario: an airborne X-band $\mathrm{M}$ SAR carried by a platform flying at $120 \mathrm{~m} / \mathrm{s}$ operates in Stripmap mode to take an image of a scene of size $2 \mathrm{~km} \times$ $100 \mathrm{~m}$ (azimuth $\times$ ground range). In this scene we simulated three-point scatterers, with the same reflecting characteristics 


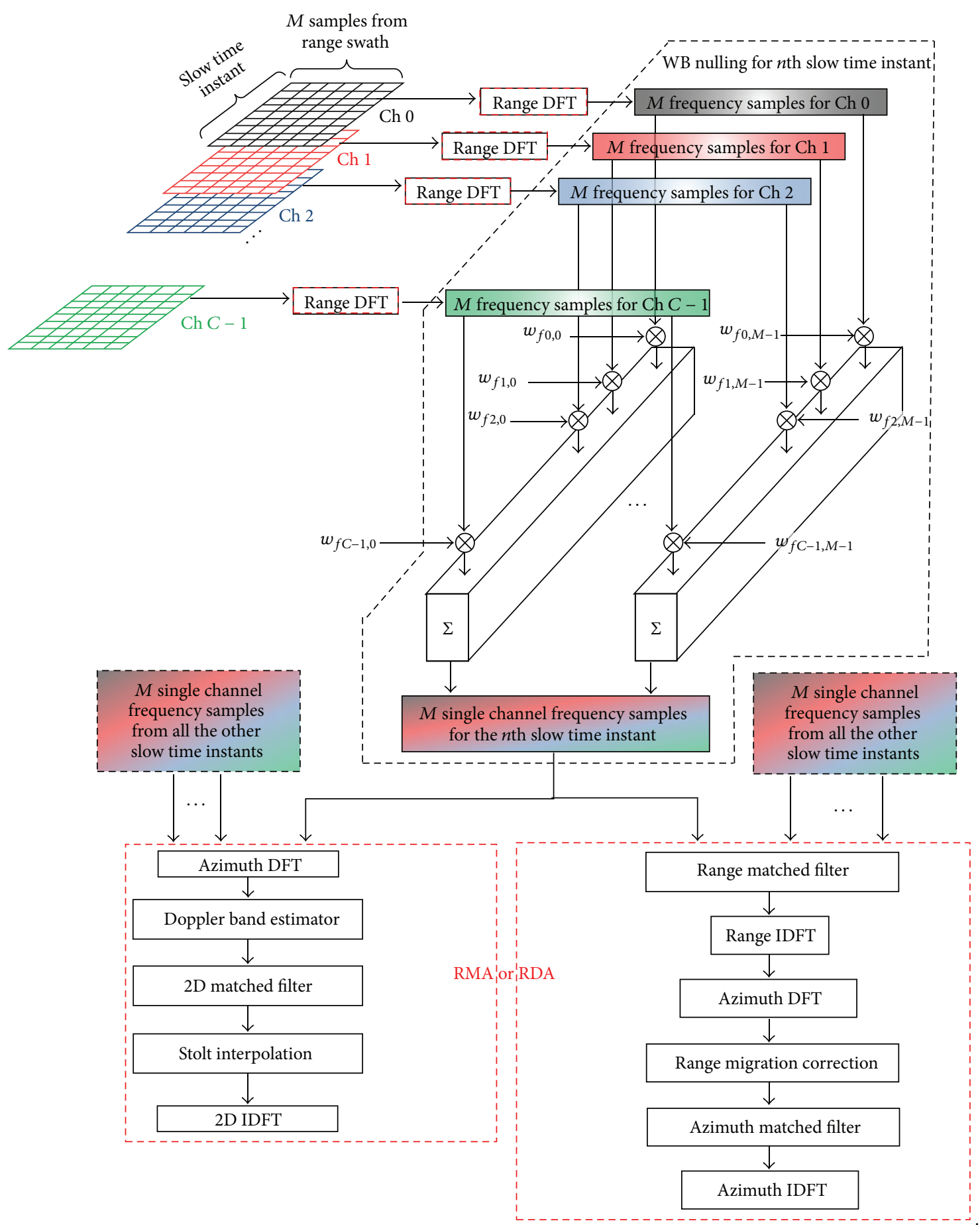

FIGURE 3: Integrated WB nulling and focusing algorithm.

but displaced in different positions in the scene: the first scatterer position is $(-900,45) \mathrm{m}$ in (azimuth, ground range), the second position is $(0,0) \mathrm{m}$, and the third scatterer position is $(900,-45) \mathrm{m}$. In order to counteract a WB interference signal, the system provides two different $r x$ channels connected at a main and an auxiliary beam. The main beam is synthesized using a phased array of size $1 \mathrm{~m} \times 0.1 \mathrm{~m}$ (azimuth $\times$ elevation); it is formed by 192 directive elements, organized in 48 elements (12 subarrays of 4 elements) in azimuth and 4 elements in elevation. The auxiliary beam is developed by a square array of 16 directive elements, 4 in the azimuth dimension and 4 in the elevation dimension with half-wavelength spacing. The main system, target, and geometry parameters are listed in Table 1.

We underline that in this case our interest is in the sidelobe noise-like wideband jammer. To this aim we simulated 
TABLE 1: Main system, target, and geometry parameters.

\begin{tabular}{lc}
\hline Carrier frequency & $9.6 \mathrm{GHz}$ \\
Transmitted power & $0.3 \mathrm{~kW}$ \\
Quiescent pattern gain & $27.7 \mathrm{~dB}$ \\
Target RCS & $3 \mathrm{~dB}$ \\
Pulse length & $5 \mu \mathrm{s}$ \\
Resolution (azimuth and range) & $0.5 \mathrm{~m}$ \\
Grazing angle & $5.7^{\circ}$ \\
Platform height & $3 \mathrm{~km}$ \\
PRF & $360 \mathrm{~Hz}$ \\
Noise figure & $5 \mathrm{~dB}$ \\
Range samples $(M)$ & 1706 \\
\hline
\end{tabular}

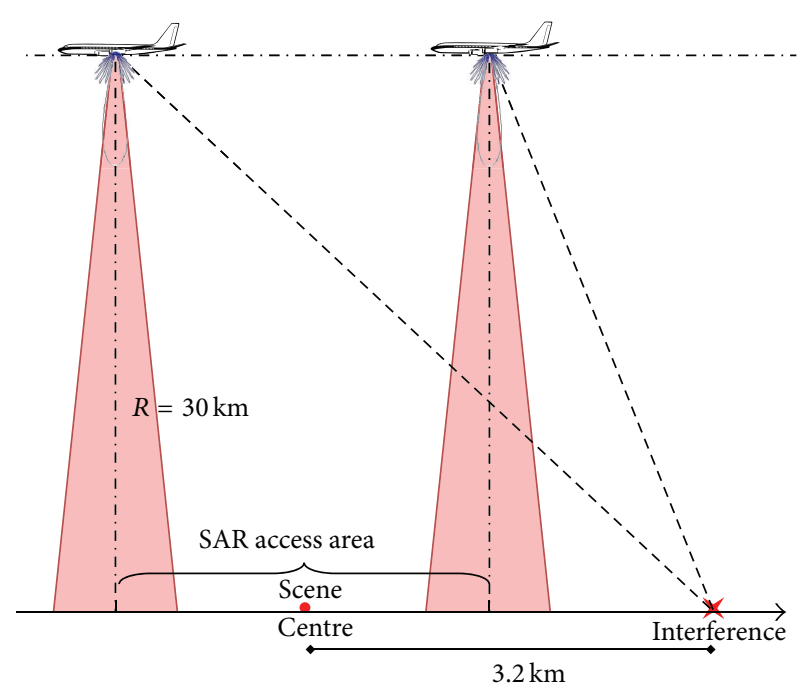

FIGURE 4: Jammer scenario.

a WB noise-like interference located at the scene center range bin displaced $3.2 \mathrm{~km}$ in along track and impinges from the second to the fourth azimuth side lobes of the main beam during the acquisition (Figure 4). We set the interference signal bandwidth matching completely the transmitted signal band and defined two jamming scenarios. They are addressed as High-Technology Jamming Scenario (HTJS) and LowTechnology Jamming Scenario (LTJS). The different level of the technology is represented by the equivalent isotropic radiated power (EIRP) value since it depends on the antenna dimensions and on the transmitted power: a jammer with an antenna of small size transmitting a low power signal (low EIRP) can disturb without the need of tracking the moving sensor, contrarily to the case of a jammer with a larger antenna transmitting a higher power. In this study we considered an EIRP of $80 \mathrm{dBW}$ for the HTJS and $50 \mathrm{dBW}$ for the LTJS. Finally RMA was used as focusing algorithm.

In order to evaluate the performance of the proposed integrated algorithm we need to define a performance parameter and reference scenario. The performance parameter is the Signal to Interference plus Noise Ratio (SINR) estimated from the image. The signal power is defined as the peak power value of the image when only the useful contribution of the

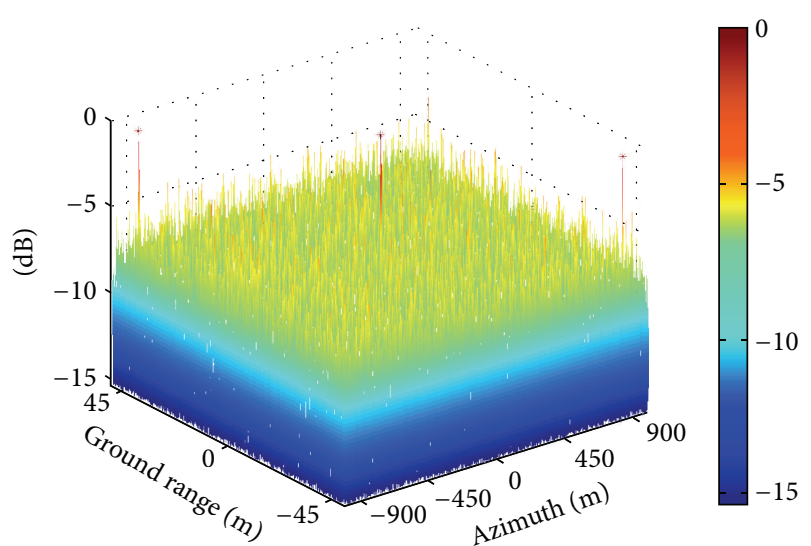

FIGURE 5: Focused image: noise and no jammer.

TABLE 2: Estimated SINR values for the HTJS and LTJS.

\begin{tabular}{lcc}
\hline Scenario/nulling technique & \multicolumn{2}{c}{ SINR (dB) } \\
& HTJS & LTJS \\
\hline Noise and no jammer & 15.3 & 15.3 \\
Noise and jammer/no ECCM & -41.15 & -11.16 \\
Noise and jammer/NB ECCM & -22.33 & 7.2 \\
Noise and jammer/WB ECCM & 15.28 & 15.28 \\
\hline
\end{tabular}

received signal is processed without adaptation, while the interference plus noise power is estimated as the mean value of the power of the disturbed image where only disturbance is present. The reference scenario is the same as the case study, but only thermal noise acts to degrade the image. Figure 5 shows the image focused with the RMA when only thermal noise is present; thus, no ECCM technique is performed; the three scatterers are visible in the image, although quite disturbed by the noise introduced, and a SINR value of $15.3 \mathrm{~dB}$ is estimated. In the following we will assume SINR values less than $10 \mathrm{~dB}$ as unable to guarantee the acquisition capability and the targets detection reliability [1]. It has to be underlined that the image in Figure 5 has been saturated to $-15.3 \mathrm{~dB}$ to allow an easier visual understanding; therefore, the noise contributions with lower amplitude level are not displaced, but they are numerically present and obviously used for the estimation of the output SINR values. Moreover, asterisks are shown in correspondence with the simulated scatterers positions to further ease the understanding of the figure, helping in identifying the scatterers peaks.

Table 2 shows the obtained values of SINR estimated from the focused images obtained with different adaptive processing; as apparent, the NB nulling algorithm is not effective against the considered WB jamming scenarios since starting from a SINR value of $-41.15 \mathrm{~dB}$ in HTJS and of $-11.16 \mathrm{~dB}$ in the LTJS if any nulling technique is applied (case addressed as noise and jammer/no ECCM), only about $19 \mathrm{~dB}$ of jammer cancellation can be achieved. In contrast, the proposed WB approach is able to almost recover the nominal SINR value, thus preserving the normal SAR operability. Figures 6 and 7 show that in both HTJS and LTJS the image is correctly focused with the three scatterers being 


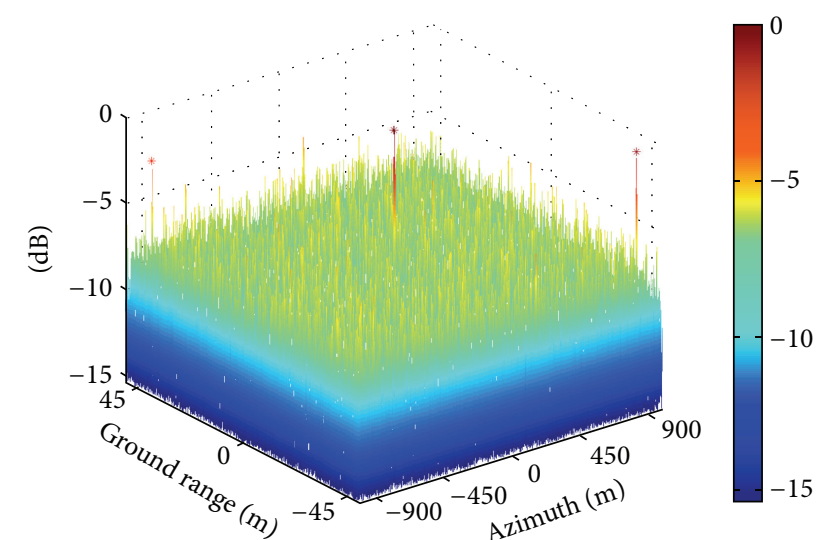

FIGURE 6: Focused image: noise and high-tech jammer/WB ECCM.

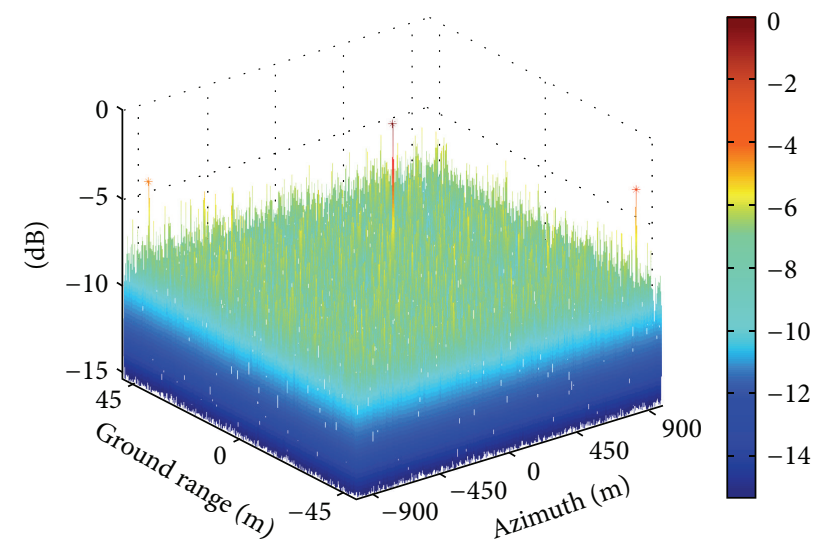

FIGURE 7: Focused image: noise and low-tech jammer/WB ECCM.

well visible above the noise level. Also in this case images have been saturated to $-15.3 \mathrm{~dB}$ for the sake of easing visual understanding.

Finally we want to evaluate the characteristics of SAR pulse response when the integrated algorithm with the wideband cancellation is performed, compared to the pulse response of the nonadapted (NA) case. To this purpose we considered the HTJS, that is, the most difficult to counteract, and consequently processed only the useful signal contribution of the received signal, not to consider the distortions introduced by the simulated noise and jammer samples. We want to underline that we proposed an integrated WB nulling and focusing algorithm mainly thinking about suppressing side-lobe noise-like jammers. Starting from this assumption we focused in a situation where we do not expect a dramatic SAR pulse response deformation, whichever the used SAR focusing algorithm, since the nulling algorithm puts nulls frequency per frequency in the adapted antenna pattern with a depth proportional to the jammer level with respect to noise in direction sufficiently distant from the pattern main lobe. This is actually the case of the simulated scenarios, where the jammer impinges from the fourth to the second sidelobe of the antenna pattern during acquisition. This is the main reason why, as apparent from Table 3, the impact on the pulse responses is limited both in range and azimuth. We can notice the increased Peak to Side-Lobe Ratio $\left(\right.$ PSLR $\left._{\mathrm{AZ}}\right)$ and the reduced azimuth resolution $\left(r_{\mathrm{AZ}}\right)$ for the nonadapted case, due to the azimuth antenna gain tapering and not to the WB nulling implementation; moreover, WB nulling tends to lower the PSLR $\mathrm{AZ}$ value with respect to the nonadapted case, depending on the characteristics of the adapted pattern synthesized to reject the interference.

Finally we can conclude that the performance in terms of SAR PSF degradation obviously depends on jammer position and power, but in the case of the considered jammer scenarios, which are the targets of our analysis, this is marginal with respect to the demonstrated cancellation capability.

\section{Analysis of the Computational Feasibility}

In the previous sections we have proved the effectiveness of the integrated algorithm to perform the WB cancellation and the focusing of an image; in this section we want to evaluate the computational burden of this technique with respect to a more traditional processing, which performs the cancellation using a WB version of the SLC [15-17] and the focusing in a sequential way (in the following addressed as sequential algorithm). RMA has been considered as focusing algorithm.

In the following subsections the cost of the integrated algorithm will be computed in terms of complex multiplications and compared with the cost of the sequential algorithm.

6.1. Cost of the Computation of the Nulling Coefficients. To null WB interferences a space-time or space-frequency weight vector has to be computed, according to (9) and (15). It is possible to identify 2 steps to compute the nulling coefficients: (i) matrix estimation and (ii) matrix inversion.

6.1.1. Matrix Estimation. It is well known that a covariance matrix can be estimated from a set of secondary signal-free data. In the case of WB frequency nulling the size of this set is $N_{\mathrm{SDF}} \geq 2 \cdot C$ to obtain losses less than $3 \mathrm{~dB}$ [30]. The $k \mathrm{th}$ spectral matrix $(k=1, \ldots, M)$ is an Hermitian matrix of size $C \times C$; therefore, the number of required complex products for the estimation of the $M$ spectral matrices is

$$
N_{\mathrm{FMAT}}=M \cdot N_{\mathrm{SDF}} \cdot \frac{C \cdot(C+1)}{2} .
$$

For the WB-SLC, the set of secondary data has size $N_{\mathrm{SDT}} \geq$ $2[1+(C-1) \cdot K]$. Due to the structure of the matrix in (10) only the first row of $\mathbf{Q}_{d \mathrm{SLC}}(n)$ and the first row of blocks of $\mathbf{Q}_{d, A}(n)$ have to be estimated (the first of this block is the block in the main diagonal and it is Hermitian). The total number of complex multiplications for the estimation can be proven equal to

$$
\begin{aligned}
& N_{\text {TMAT }} \\
& =N_{\mathrm{SDT}} \\
& \quad \cdot\left[(K-1) \cdot(C-1)^{2}+\left(\frac{C}{2}+K\right) \cdot(C-1)+1\right] .
\end{aligned}
$$


TABLE 3: SAR pulse response quality parameters for the HTJS.

\begin{tabular}{|c|c|c|c|c|c|c|}
\hline \multirow[t]{2}{*}{ Scatterer position } & \multicolumn{2}{|c|}{$\begin{array}{l}\text { First scatterer } \\
(-45,900) \mathrm{m}\end{array}$} & \multicolumn{2}{|c|}{$\begin{array}{c}\text { Second scatterer } \\
(0,0) \mathrm{m}\end{array}$} & \multicolumn{2}{|c|}{$\begin{array}{l}\text { Third scatterer } \\
(45,-900) \mathrm{m}\end{array}$} \\
\hline & NA & WB ECCM & NA & WB ECCM & NA & WB ECCM \\
\hline$r_{\mathrm{AZ}}(\mathrm{m})$ & 0.56 & 0.56 & 0.56 & 0.56 & 0.56 & 0.56 \\
\hline $\operatorname{PSLR}_{\mathrm{AZ}}(\mathrm{dB})$ & 18.32 & 17.21 & 18.33 & 17.14 & 18.35 & 17.29 \\
\hline$r_{\mathrm{RG}}(\mathrm{m})$ & 0.51 & 0.51 & 0.505 & 0.51 & 0.51 & 0.51 \\
\hline $\operatorname{PSLR}_{\mathrm{RG}}(\mathrm{dB})$ & 12.95 & 12.95 & 13.12 & 13.12 & 13.25 & 13.24 \\
\hline
\end{tabular}

6.1.2. Matrix Inversion and Computation of the Coefficients. In both the frequency and time nulling techniques, the weight vector is the first column of the inverse of the $k$ th spectral or covariance matrix in (13) or (10), respectively. For the frequency WB nulling, it can be proven to depend only on the inverse of the spectral covariance matrix pertaining to the auxiliary channels $\mathbf{Q}_{d f, A}(n, k)$ of size $(C-1) \times(C-1)$ and the vector $\mathbf{q}_{d f, M A}(n, k)$ of size $1 \times(C-1)$ describing the covariance between the main and the auxiliary channels in the frequency domain. Therefore, the total number of complex products required in this case is

$$
N_{\mathrm{FW}}=M \cdot(C-1)^{2} \cdot[(C-1)+1] .
$$

For the WB-SLC the weight vector can be proven to depend only on $\mathbf{q}_{d, M A}(n)$ and on the inverse of $\mathbf{Q}_{d, A}(n)$. The total number of complex products in this case is

$$
N_{\mathrm{TW}}=[(C-1) \cdot K]^{2} \cdot[(C-1) \cdot K+1] .
$$

6.2. Computational Cost of the WB Nulling Techniques. For all of the proposed techniques a set of multichannel data has to be filtered to obtain single-channel data and several (or one in the WB-SLC case) FFT have to be performed to efficiently implement the required DFTs. As it is well known, to compute the single FFT over a number of $M$ points (if $M$ is a power of 2 ), the total number of required complex products is

$$
N_{\mathrm{FFT}}=\frac{M}{2} \cdot \log _{2}(M) \text {. }
$$

Moreover, for the WB nulling in the frequency domain, a number $C$ of FFTs and a number $M$ of complex products have to be performed for each channel to feed the subsequent focusing algorithm. For the WB-SLC case, for all the $M$ fasttime samples $K$ products between complex numbers have to be performed for each auxiliary channel and only one for the main channel; the obtained equivalent single-channel data needs then to be Fourier transformed to be a suitable input for the following RMA. Therefore, the total number of complex products per PRT for nulling is

$$
\begin{aligned}
& N_{\text {FWBnull }}=C \cdot\left(\frac{M}{2} \cdot \log _{2}(M)+M\right), \\
& N_{\text {TWBnull }}=\frac{M}{2} \cdot \log _{2}(M)+[1+(C-1) \cdot K] \cdot M
\end{aligned}
$$

in the frequency and in the time domain, respectively.
In our study case it is possible to verify that independently of the number of receiving channels $C$ considered, for $K>7$, the technique in the frequency domain is "cheaper" independently of the number of receiving channels; see Figure 8.

6.3. Computational Cost of the Range Migration Algorithm. The RMA procedure consists in (i) pass-band filtering to reduce the number of samples to be processed in the azimuth domain from $N$ to $N_{P}$, (ii) $2 \mathrm{D}$ matched filtering with a cost of $M \cdot N_{P}$ complex multiplications, and (iii) Stolt interpolation. The implementation of this last step takes account of $N$ iterations of the interpolation cycle, for which $N_{Y}$ iterations are performed. $N_{Y}$ represents the number of samples of the new range axis over which the original range axis is mapped by the Stolt interpolation. The interpolation filter used has $I$ taps. Therefore, the total number of complex multiplications for the RMA can be proven equal to

$$
\begin{aligned}
N_{\mathrm{RMA}} & =N \cdot M \\
\cdot & {\left[\frac{1}{2} \log _{2}\left(N^{\left(N_{Y} / N\right)+1} \cdot\left(M \cdot \frac{N_{Y}}{N}\right)^{N_{Y} N_{P} / N^{2}}\right)+\frac{N_{P}}{N}\right.} \\
+ & \left.\left(\frac{N_{Y}}{N}\right) \cdot 4 \cdot I\right] .
\end{aligned}
$$

6.4. Computational Cost Comparison. Figure 9 shows the comparison between the cost of the RMA and the overall cost of the time domain and frequency domain WB nulling techniques, $N_{F}$ and $N_{T}$, respectively:

$$
\begin{aligned}
& N_{F}=\left[N_{\text {FWBnull }}+N_{\text {FMAT }}+N_{\text {FW }}\right] \cdot N, \\
& N_{T}=\left[N_{\text {TWBnull }}+N_{\text {TMAT }}+N_{\text {TW }}\right] \cdot N .
\end{aligned}
$$

As apparent, in the case study considered the load of the focusing algorithm is lower than the cost of the other operations. Moreover, we can see that it is possible to find a value of $K$, consistent with the length of the fast-time filters needed by the WB-SLC as the bandwidth and the EIRP of the jammer increases [29], which allows the total load of the WB nulling in the frequency domain to be comparable with the total load of the WB-SLC. 
TABLE 4: Suboptimal WB approach in the range dimension: SINR versus $N_{s}$.

\begin{tabular}{|c|c|c|c|c|c|c|c|c|c|c|c|c|c|}
\hline$N_{s}$ & & 1 & 2 & 3 & 4 & 5 & 8 & 16 & 32 & 64 & 128 & 246 & 1706 \\
\hline \multirow{2}{*}{$\operatorname{SINR}(\mathrm{dB})$} & HTJS & -22.3 & -16.3 & - & -10.3 & - & -4.4 & 1.4 & 7.05 & 11.3 & 14.0 & 14.7 & 15.28 \\
\hline & LTJS & 7.2 & 11.5 & 13.2 & 14.0 & 14.4 & 14.9 & 15.1 & - & - & - & - & 15.28 \\
\hline
\end{tabular}

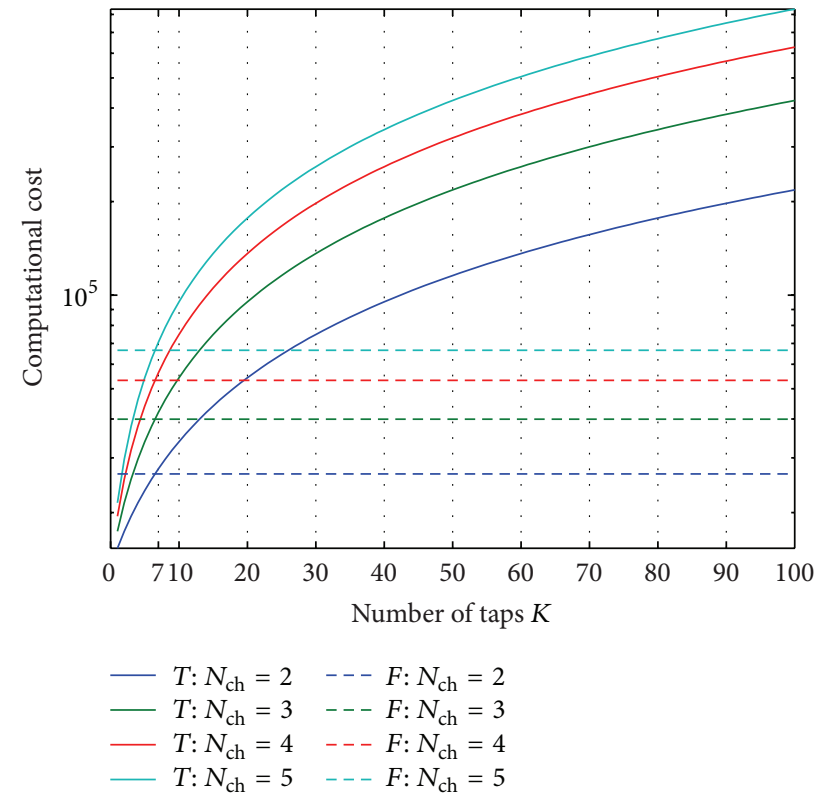

FIGURE 8: Computational cost of the WB-SLC (labelled with $T$ in the legend) for different values of $K$ and $C$, compared with the values of the computational cost of the WB frequency nulling SLC (labelled with $F$ in the legend) for the same value of $C$.

\section{Suboptimal Implementation of the Space- Frequency Adaptive Nulling Algorithm}

To reduce the computational burden required by the spacefrequency adaptive WB nulling technique, suboptimal (SO) implementations are now considered in contrast with the optimal WB nulling (OPT) described in Section 4. This could be particularly useful when an estimate of the spectral covariance matrices has to be provided from secondary data. Given that the matrix estimation has to be performed for each frequency subband every specified number of PRT, the suboptimal approach allows reducing the size of secondary data required. The case of a very limited availability of targetfree secondary data might be of special interest for SAR systems where the echoes from the area of interest tend to occupy almost all the available reception window.

7.1. Suboptimal Implementation in the Range Dimension. In the proposed SO-WB nulling technique the whole signal band is divided into $N_{s}$ subbands $\left(1 \leq N_{s} \leq M\right)$ and a single weight vector is applied to all the spectral components within the frequency subband. The achieved SINR values versus $N_{s}$ are reported in Table 4 for both HTJS and LTJS. As apparent, when a strong jammer impinges on the antenna a reduced number of 256 subbands, that is, a reduction of a

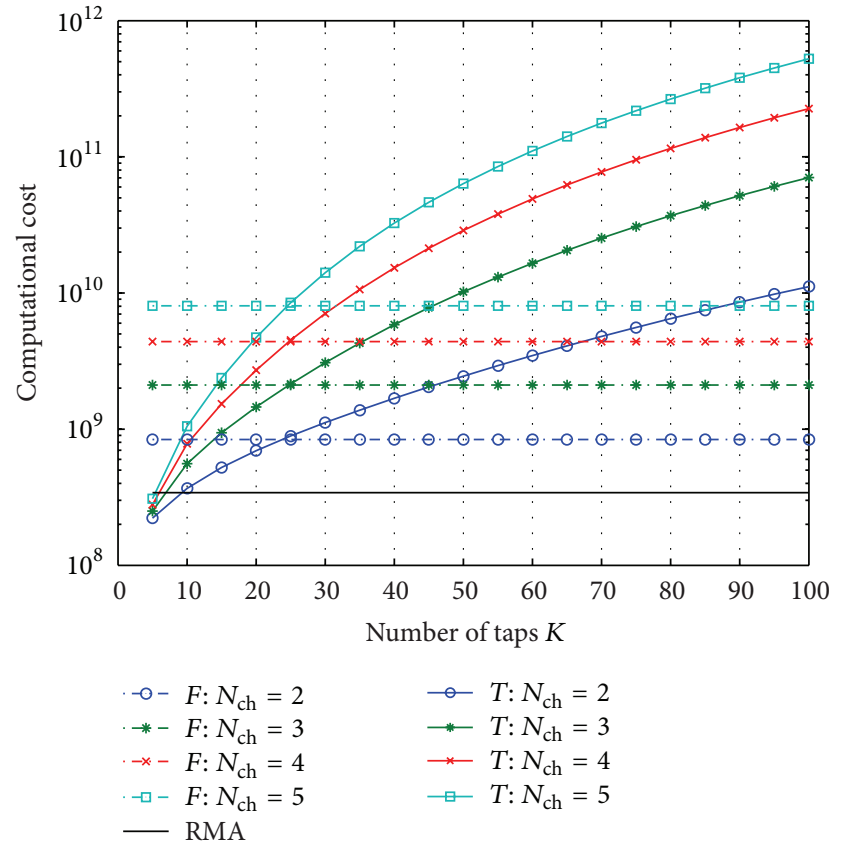

FIGURE 9: Comparison of the overall computational cost of the RMA and the WB nulling techniques in the time and in the frequency domain.

factor 7 in the computational cost for the evaluation of the nulling weights, allow us to almost recover the performance of the optimal WB technique with losses lower than $1 \mathrm{~dB}$; the same result is obtained using 5 subbands for the LTJS, with a reduction of a factor 340 in the computational cost for the evaluation of the nulling weights.

The proposed nulling technique performs a filtering operation of the received signal in the fast-time dimension; this could lead to a deformation of the range pulse response. To confirm and improve the results shown in Table 3 in the case of a HTJS, that is, the most difficult to counteract, Figure 10 shows how no deformations are experienced, even when a strong amplitude tapering function (i.e., Hamming) is applied in range to the useful signal. As apparent, the paired echoes arisen from filtering determine a slight increase of the first side-lobes, however about $40 \mathrm{~dB}$ below the target peak value. We recall that both in Section 5 and in here the analysis of the PSF characteristics has been performed processing only the useful component of the SAR signal, therefore without jammer and noise, to show the effect of the processing not affected by the actual amount of achieved jammer cancellation.

7.2. Suboptimal Implementation in the Azimuth Dimension. In the proposed $\mathrm{WB}$ nulling technique in the frequency 
TABLE 5: Suboptimal WB approach in the azimuth dimension: SINR versus $N_{u}$ and the block size.

\begin{tabular}{|c|c|c|c|c|c|c|c|c|c|c|}
\hline \multirow{3}{*}{ HTJS } & \multicolumn{2}{|c|}{$N_{u}$ (block size $)$} & $383(23)$ & 464 (19) & $588(15)$ & $801(11)$ & $1260(7)$ & $1763(5)$ & $2939(3)$ & $8816(1)$ \\
\hline & \multirow{2}{*}{ SINR (dB) } & Central pulse & 0.03 & 1.61 & 3.58 & 6.03 & 9.31 & 11.33 & 13.54 & 15.28 \\
\hline & & First pulse & -5.59 & -3.91 & -1.78 & 0.98 & 5.02 & 7.93 & 11.79 & 15.28 \\
\hline \multirow{2}{*}{ LTJS } & \multicolumn{2}{|c|}{$N_{u}$ (block size $)$} & 3 (2939) & $9(979)$ & $15(587)$ & 45 (195) & $93(95)$ & $173(51)$ & $285(31)$ & $464(19)$ \\
\hline & SINR (dB) & Central pulse & -9.51 & $-2-27$ & 1.90 & 10.06 & 13.36 & 14.64 & 15.03 & 15.18 \\
\hline
\end{tabular}

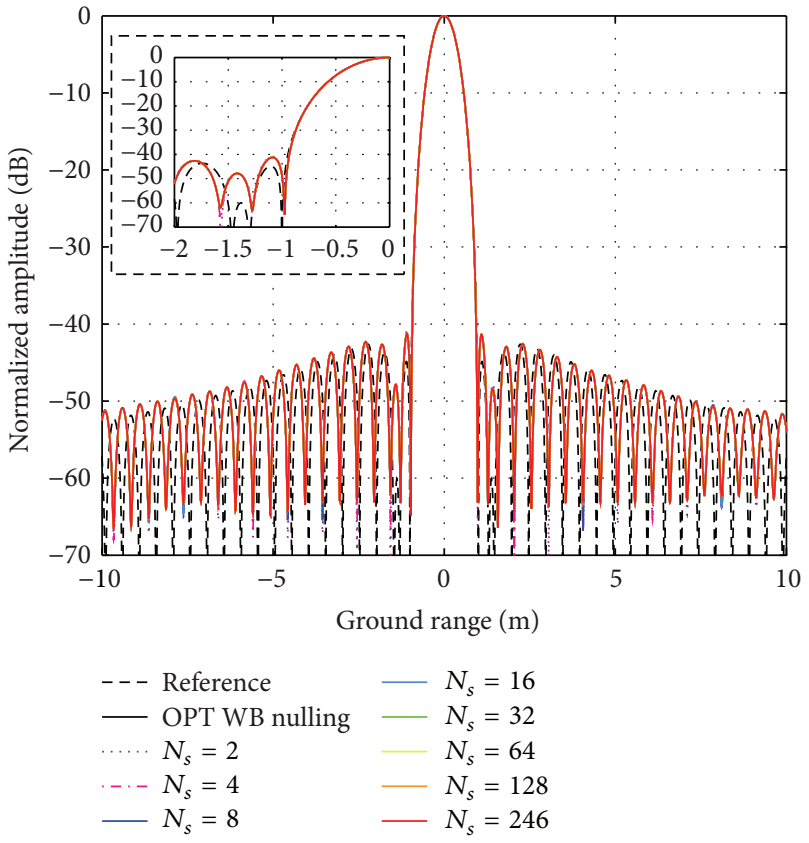

FIGURE 10: Range pulse response with Hamming tapering (HTJS).

domain the nulling coefficients are varying in the slow-time; for each slow-time pulse during the acquisition the weight vector applied to null the interference changes, changing the DoA of the interference with respect to the SAR antenna. This implies that theoretically the nulling coefficients have to be updated for each slow-time pulse.

The suboptimal approach in the azimuth dimension aims at reducing the number updates of the nulling weight vector. In this case the total number of slow-time pulses $N$ is divided into $N_{u}$ blocks $\left(1 \leq N_{u} \leq N\right)$ and the updating process is realized $N_{u}$ times instead of $N$. For a HTJS we considered the possibility of computing the nulling coefficients in correspondence of the first or of the central pulse in each block and then used them for all the pulses in the block. As apparent from Table 5 updating the set of coefficients at the first slow time pulse in each block is worse than at the central slow-time pulse. Moreover, we can see that the number of updates that guarantees reasonable performance is slightly less than $N$; as said before the direction of arrival of the interference varies during the acquisition and the use of the same set of coefficients to null adjacent directions seems to be ineffective. This behavior seems to suggest that the nulling procedure is more sensitive to the slow-time variations than to the fast-time variations of the nulling coefficients, thus yielding relevant performance
TABLE 6: Suboptimal WB approach both in the azimuth and in the range dimension: SINR (dB) versus $N_{u}$ and $N_{s}$ for the HTJS and the LTJS.

\begin{tabular}{ccccccccccc}
\hline & \multicolumn{3}{c}{ HTJS } & & \multicolumn{5}{c}{ LTJS } \\
& & \multicolumn{3}{c}{$N_{u}$} & & & & & $N_{u}$ & \\
& & 801 & 1260 & 2939 & & & 464 & 93 & 45 \\
\hline \multirow{2}{*}{$N_{s}$} & 32 & 3.89 & 5.6 & 6.74 & & 2 & 11.51 & 10.67 & 8.68 \\
& 64 & 5.46 & 7.97 & 10.57 & $N_{s}$ & 3 & 13.15 & 11.95 & 9.41 \\
& 128 & 6.00 & 9.01 & 12.7 & & 8 & 14.81 & 13.16 & 10.05 \\
\hline
\end{tabular}

degradation when the suboptimal nulling approach is applied in the azimuth dimension with respect to the suboptimal nulling approach in the range dimension. It has to be said that this is closely related to the characteristics of the WB interference signal, especially in terms of EIRP; reasonably a lower value of EIRP could allow reducing the performance degradation when the suboptimal nulling in the azimuth dimension is performed. This is confirmed by the study of the LTJS; as apparent from Table 5, 173 updates over 8816, that is, a block size of 51 slow-time pulses, guarantee reasonable performance, with losses lower than $1 \mathrm{~dB}$.

7.3. Suboptimal Implementation Both in the Azimuth and in the Range Dimension. To further reduce the computational cost it is possible to implement a suboptimal nulling both in azimuth and in range. Generally we can observe that the achievable performance in terms of SINR has a higher bound that is the lower value between the SINRs obtained with the suboptimal approach in the range and in the azimuth dimension separately. Results for the HTJS and LTJS are listed in Table 6.

\section{Conclusions}

In this paper we considered a WB antenna nulling technique based on a space-frequency adaptive nulling integrated within the RMA focusing algorithm for M-SAR systems, allowing a fusion between ECCM and usual SAR processing steps. Performance analysis showed the effectiveness of the proposed integrated approach in terms of interference rejection and SAR point spread function characteristics preservation. The computational cost of the integrated algorithm has been compared in detail with the cost of more traditional sequential algorithm based on WB-SLC, showing the computational feasibility of the proposed integrated algorithm, especially under severe interference conditions when the length of the fast-time filters of the WB-SLC increases. To 
reduce the computational cost of the integrated algorithm, the possibility to suboptimally perform the space-frequency adaptive processing has been considered; the WB nulling performance showed to be more affected by a reduction in the number of slow-time updates of the nulling coefficients than the in number of considered subbands.

\section{Conflict of Interests}

The authors declare that there is no conflict of interests regarding the publication of this paper.

\section{References}

[1] W. W. Goj, Synthetic Aperture Radar and Electronic Warfare, Artech House, Norwood, Mass, USA, 1989.

[2] M. Sedehi, D. Cristallini, J. Marini, and P. Lombardo, "Impact of an electromagnetic interference on imaging capability of a synthetic aperture radar," in Proceedings of the IEEE Aerospace Conference, pp. 1-8, Big Sky, Mont, USA, March 2007.

[3] K. Dumper, P. S. Cooper, A. F. Wons, C. J. Condley, and P. Tully, Spaceborne Synthetic Aperture Radar and Noise Jamming, Systems Engineering \& Assessment, Bristol, UK, 1997.

[4] A. Farina, Antenna-Based Signal Processing Techniques for Radar Systems, Artech House, Boston, Mass, USA, 1992.

[5] M. Skolnik, Radar Handbook, Edited by J. Frank and J. D. Richards, chapter 13, McGraw-Hill, New York, NY, USA, 2008.

[6] P. Lombardo, R. Cardinali, M. Bucciarelli, D. Pastina, and A. Farina, "Planar thinned arrays: optimization and subarray based adaptive processing," International Journal of Antennas and Propagation, vol. 2013, Article ID 206173, 13 pages, 2013.

[7] P. Lombardo, R. Cardinali, D. Pastina, M. Bucciarelli, and A. Farina, "Array optimization and adaptive processing for subarray based thinned arrays," in Proceedings of the International Conference on Radar (Radar '08), pp. 197-202, IEEE, Adelaide, Australia, September 2008.

[8] R. T. Compton Jr., Adaptive Antennas Concepts and Performance, Prentice Hall, New York, NY, USA, 1988.

[9] H. Steyskal, "Wide-band nulling performance versus number of pattern constraints for an array antenna," IEEE Transactions on Antennas and Propagation, vol. 31, no. 1, pp. 159-163, 1983.

[10] B. Widrow, P. E. Mantey, L. J. Griffiths, and B. Goode, "Adaptive antenna systems," Proceedings of the IEEE, vol. 55, no. 12, pp. 2143-2159, 1967.

[11] J. T. Mayhan, A. J. Simmons, and W. C. Cummings, "Wideband adaptive antenna nulling using Tapped Delay-Lines," IEEE Transaction on Antennas and Propagation, vol. 29, no. 6, 1981.

[12] Q. Zou, Z. L. Yu, and Z. Lin, "A robust algorithm for linearly constrained adaptive beamforming," IEEE Signal Processing Letters, vol. 11, no. 1, pp. 26-29, 2004.

[13] Y. Zhao, W. Liu, and R. J. Langley, "Adaptive wideband beamforming with frequency invariance constraints," IEEE Transactions on Antennas and Propagation, vol. 59, no. 4, pp. 1175-1184, 2011.

[14] B. K. Mathai, S. Gopi, and R. Pradeepa, "A recursive algorithm for robust wideband adaptive beamforming," in Proceedings of the 2nd International Conference on Communication and Signal Processing (ICCSP '13), pp. 1043-1047, Melmaruvathur, India, April 2013.
[15] L. Rosenberg and D. A. Gray, "Constrained fast-time STAP for interference suppression in multichannel SAR," IEEE Transactions on Aerospace and Electronic Systems, vol. 49, no. 3, pp. 1792-1805, 2013.

[16] W. D. White, "Wideband interference cancellation in adaptive sidelobe cancellers," IEEE Transactions on Aerospace and Electronic Systems, vol. 19, no. 6, pp. 915-925, 1983.

[17] R. L. Fante, R. M. Davis, and T. P. Guella, "Wideband cancellation of multiple mainbeam jammers," IEEE Transactions on Antennas and Propagation, vol. 44, no. 10, pp. 1402-1413, 1996.

[18] R. T. Compton Jr., "The relationship between Tapped DelayLine and FFT processing in adaptive arrays," IEEE Transactions on Antennas and Propagation, vol. 36, no. 1, pp. 15-26, 1988.

[19] B. Himed, K. Kim, Y. Zhang, and A. Hajjari, "A new approach to wideband space-time adaptive processing (W-STAP)," in Proceedings of the Sensor Array and Multichannel Signal Processing Workshop, pp. 672-676, Barcelona, Spain, July 2004.

[20] S. U. Pillai, K. Y. Li, and J. R. Guerci, "Efficient wideband processing without subbanding," in Proceedings of the IEEE Radar Conference (RADAR '08), pp. 1-5, IEEE, Rome, Italy, May 2008.

[21] W.-T. Lin and K.-B. Yu, "Adaptive beamforming for wideband jamming cancellation," in Proceedings of the IEEE National Radar Conference, pp. 82-87, Syracuse, NY, USA, May 1997.

[22] J. A. Torres, R. M. Davis, J. D. R. Kramer, and R. L. Fante, "Efficient wideband jammer nulling when using stretch processing," IEEE Transactions on Aerospace and Electronic Systems, vol. 36, no. 4, pp. 1167-1178, 2000.

[23] Y. Cao, S. Zhang, H. Wang, and Z. Gao, "Wideband adaptive sidelobe cancellation based on stretch processing," in Proceedings of the 8th International Conference on Signal Processing (ICSP '06), Beijing, China, November 2006.

[24] H. Mir and Z. Berkowitz, "Sub-band stap for stretch processed systems," in Proceedings of the IEEE International Conference on Acoustics, Speech, and Signal Processing (ICASSP '09), pp. 20252028, Taipei, Taiwan, April 2009.

[25] C. Cafforio, C. Prati, and F. Rocca, "SAR data focusing using seismic migration techniques," IEEE Transactions on Aerospace and Electronic Systems, vol. 27, no. 2, pp. 194-207, 1991.

[26] J. C. Curlander and R. N. McDonough, Synthetic Aperture Radar. Systems and Signal Processing, John Wiley \& Sons, 1991.

[27] M. Bucciarelli, D. Cristallini, D. Pastina, M. Sedehi, and P. Lombardo, "Integrated wideband antenna nulling and focusing technique for Multi-Channel Synthetic Aperture Radar," in Proceedings of the International Radar Symposium (IRS '08), pp. 1-4, Wroclaw, Poland, May 2008.

[28] J. H. G. Ender, "Space-time processing for multichannel synthetic aperture radar," Electronics \& Communication Engineering Journal, vol. 11, no. 1, pp. 29-38, 1999.

[29] M. Bucciarelli, M. Sedehi, and P. Lombardo, "Wideband adaptive antenna nulling schemes for Synthetic Aperture Radar," in Proceedings of the IEEE Radar Conference (RADAR '08), pp. 1-6, Rome, Italy, May 2008.

[30] I. S. Reed, J. D. Mallett, and L. E. Brennan, "Rapid convergence rate in adaptive arrays," IEEE Transactions on Aerospace and Electronic Systems, vol. 10, no. 6, pp. 853-863, 1974. 


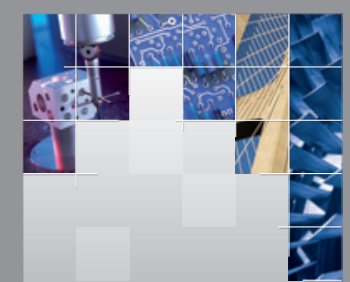

\section{Enfincering}
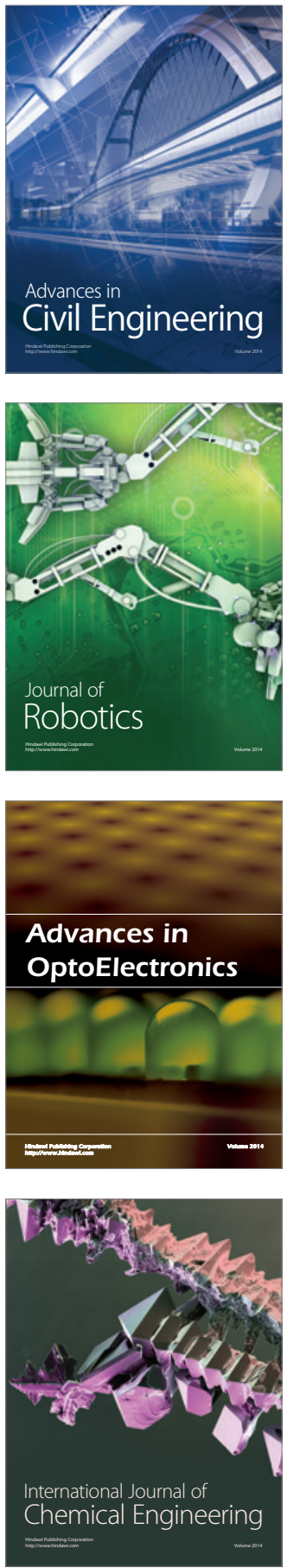

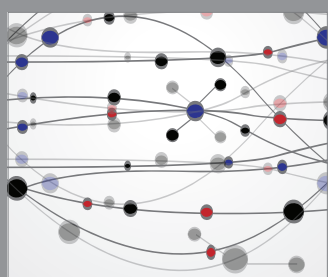

The Scientific World Journal

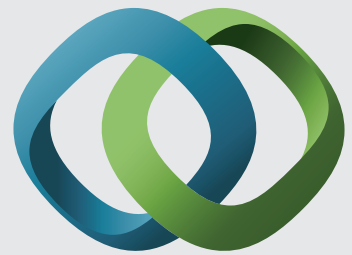

\section{Hindawi}

Submit your manuscripts at

http://www.hindawi.com
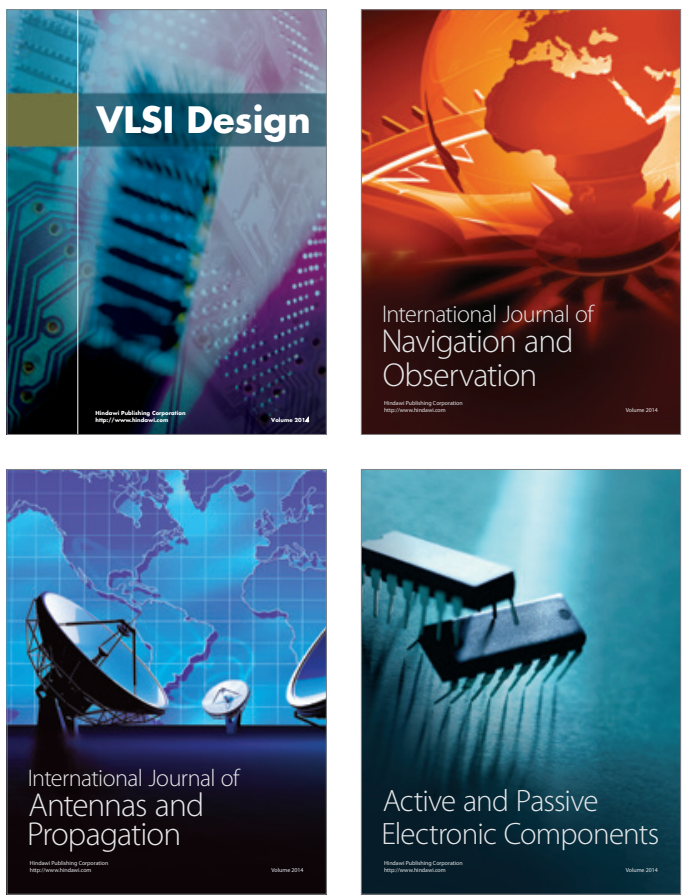
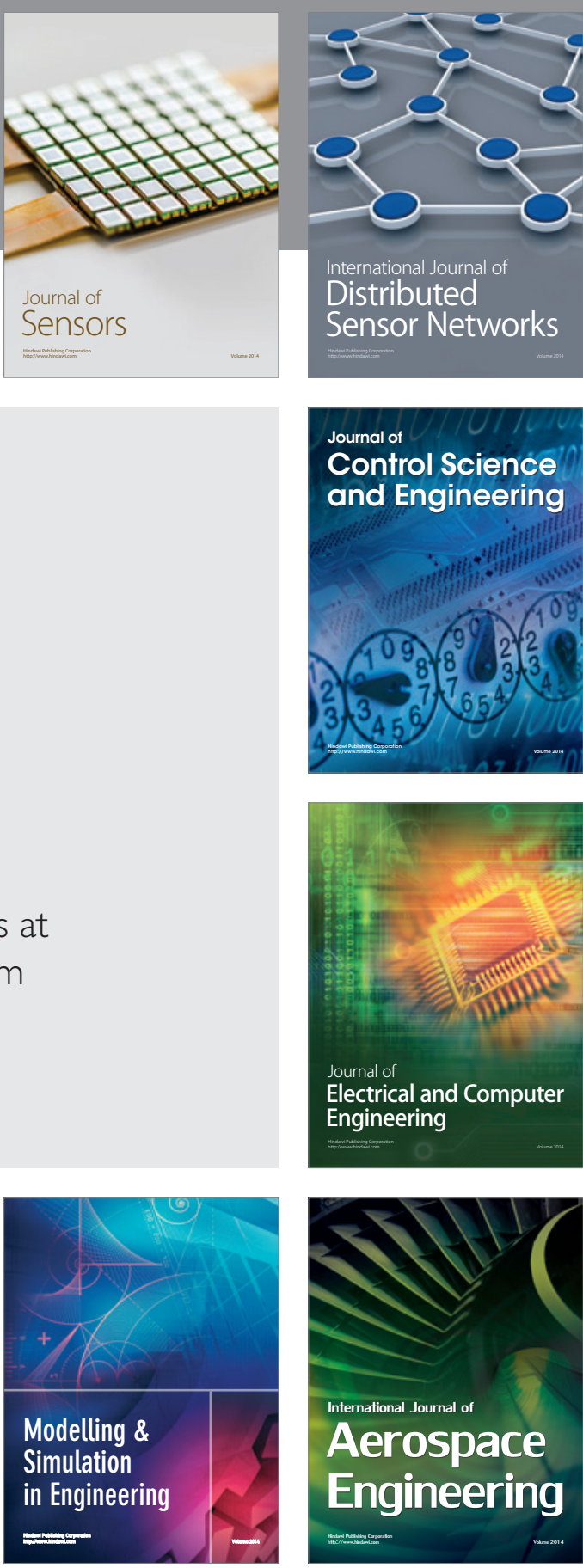

International Journal of

Distributed

Sensor Networks

Journal of

Control Science

and Engineering
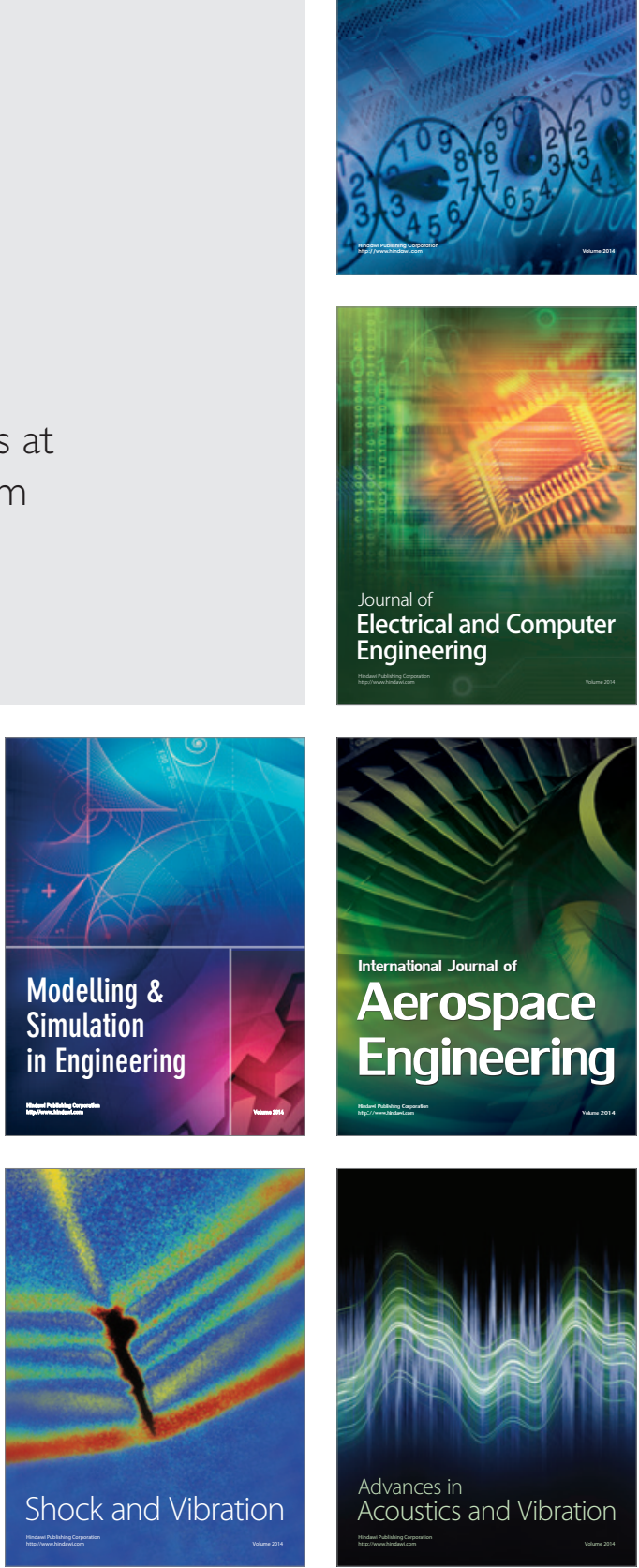